BMJ Open

Diabetes

Research

\& Care

\section{Microscopic hematuria is a risk factor for end-stage kidney disease in patients with biopsy-proven diabetic nephropathy}

To cite: Okada S, Samejima K, Matsui M, et al. Microscopic hematuria is a risk factor for end-stage kidney disease in patients with biopsyproven diabetic nephropathy. BMJ Open Diab Res Care 2020;8:e001863. doi:10.1136/ bmjdrc-2020-001863

SO and K-iS contributed equally.

Received 26 August 2020 Revised 12 September 2020 Accepted 17 September 2020

Check for updates

(c) Author(s) (or their employer(s)) 2020. Re-use permitted under CC BY-NC. No commercial re-use. See rights and permissions. Published by BMJ.

${ }^{1}$ Center for Postgraduate Training, Nara Medical University, Kashihara, Nara, Japan

${ }^{2}$ Department of Diabetes and Endocrinology, Nara Medical University, Kashihara, Nara, Japan

${ }^{3}$ Department of Nephrology, Nara Medical University, Kashihara, Nara, Japan ${ }^{4}$ Department of Nephrology, Nara Prefecture General Medical Center, Nara, Japan ${ }^{5}$ Department of Nephrology, Nara Prefecture Seiwa Medical Center, Nara, Japan

${ }^{6}$ Department of Cardiology, Nara Medical University, Kashihara, Nara, Japan

Correspondence to Dr Ken-ichi Samejima; ksame@naramed-u.ac.jp

\section{ABSTRACT}

Introduction There are fewer reports about whether the presence of hematuria affects the progression of chronic kidney disease in patients with diabetic nephropathy. We analyzed whether microscopic hematuria in diabetic nephropathy is a risk factor for end-stage kidney disease (ESKD).

Research design and methods The present study was a retrospective cohort study of patients with biopsy-proven diabetic nephropathy. We recruited 397 patients with diabetic nephropathy, which was confirmed by renal biopsy between June 1981 and December 2014 and followed them until October 2018 or death. Patients with microscopic hematuria before renal biopsy were defined as the hematuria group $(n=91)$, and the remainder as the no-hematuria group $(n=306)$. The main outcome was the occurrence of ESKD, which was defined by the requirement of permanent renal replacement therapies.

Results The systolic and diastolic blood pressure, serum creatinine and proteinuria were significantly higher, and the estimated glomerular filtration rate was significantly lower in the hematuria group compared with the no-hematuria group. Pathological evaluations revealed that glomerular, tubulointerstitial and vascular lesions in the hematuria group were significantly more severe. During a median of 10.1 years, 44 and 52 patients developed ESKD in the hematuria group and the no-hematuria group, respectively. Survival analyses showed that the incidence of ESKD was significantly higher in the hematuria group compared with the no-hematuria group (log-rank, $p<0.0001)$. The multivariable Cox proportional hazards models revealed a significant association between hematuria and the incidence of ESKD after adjusting for clinically relevant factors, including proteinuria and renal pathology (adjusted HR $1.64,95 \% \mathrm{Cl} 1.03$ to 2.60). The subgroups of men, proteinuria $\geq 0.5 \mathrm{~g} /$ day, and systolic blood pressure $\geq 132 \mathrm{~mm} \mathrm{Hg}$ showed a stronger association between hematuria and ESKD than their opposing subgroups. Conclusions Microscopic hematuria is a risk factor for ESKD in diabetic nephropathy, independent of proteinuria and renal pathology.

\section{INTRODUCTION}

Glomerular hematuria has been classically considered a hallmark of glomerular filtration barrier dysfunction or damage, but with less effect on the progressive decline in renal

\section{Significance of this study}

What is already known about this subject?

- The presence of hematuria has been considered a clinical sign of non-diabetic kidney disease in patients with diabetes.

- There are fewer reports about whether microscopic hematuria affects the progression of chronic kidney disease in patients with diabetic nephropathy.

What are the new findings?

The presence of microscopic hematuria is a risk factor for end-stage kidney disease (ESKD) in diabetic nephropathy, independent of proteinuria and renal pathology.

How might these results change the focus of research or clinical practice?

- We should treat patients with diabetic nephropathy and microscopic hematuria as high-risk patients for ESKD.

function. ${ }^{1}$ Recently, several studies reported that the presence of hematuria was associated with a faster decline in renal function and an increase in development of end-stage kidney disease (ESKD) in healthy people ${ }^{2} 3$ and patients with chronic kidney disease (CKD). ${ }^{4}$ Although diabetic nephropathy is the leading cause of ESKD, the clinical assessment of diabetic nephropathy includes the level of albuminuria and estimated glomerular filtration rate (eGFR), and it does not consider the presence of hematuria. ${ }^{5}$ The presence of hematuria has been considered a clinical sign of non-diabetic kidney disease in patients with diabetes ${ }^{6-8}$; however, a recent report of biopsy-proven diabetic nephropathy showed that patients with advanced diabetic nephropathy were accompanied by a high prevalence of hematuria. ${ }^{9}$ There are fewer reports about whether the presence of hematuria affects the progression of CKD in patients with diabetic nephropathy. 
A previous study reported that patients with hematuria had more advanced glomerular and vascular lesions in renal pathology than those without hematuria. ${ }^{10}$ Therefore, we hypothesized that glomerular hematuria in diabetic nephropathy is a risk factor for the progression of CKD. In the present study, we assessed whether the presence of microscopic hematuria was associated with the incidence of ESKD in patients with biopsy-proven diabetic nephropathy.

\section{RESEARCH DESIGN AND METHODS \\ Study design}

This study was a retrospective cohort study of patients with biopsy-proven diabetic nephropathy. ${ }^{11}$ Informed consent was obtained from each patient.

\section{Study population}

A total of 408 patients with type 2 diabetes, who were proven to have diabetic nephropathy by renal biopsy at Nara Medical University Hospital between June 1981 to December 2014, completed the follow-up study. We excluded 11 patients whose prerenal biopsy urinary sediment data were missing. Eventually, we followed 397 patients until October 2018 or death.

The following clinical data were obtained at the renal biopsy: age, sex, body mass index (BMI), smoking status, presence of diabetic retinopathy, systolic and diastolic blood pressure, serum creatinine, hemoglobin Alc (HbA1c), proteinuria and hematuria. Serum creatinine values $(\mathrm{mg} / \mathrm{dL})$ measured by the Jaffe method were converted to values for the enzymatic method by subtracting $0.207 \mathrm{mg} / \mathrm{dL}{ }^{12}$ The eGFR $\left(\mathrm{mL} / \mathrm{min} / 1.73 \mathrm{~m}^{2}\right.$ of body surface area) was calculated using the new threevariable Japanese equation for eGFR: eGFR=194×serum creatinine $(\mathrm{mg} / \mathrm{dL})^{-1.094} \times$ age $^{-0.287} \times 0.739$ (if female). ${ }^{13}$ HbAlc values measured by the Japan Diabetes Society values were converted to the National Glycohemoglobin Standardization Program values. ${ }^{14}$ Proteinuria was measured by 24-hour urine protein (or spot urine protein creatinine ratio, if 24-hour urine protein was not available).

\section{Exposure}

In the present study, we analyzed the association between the prevalence of hematuria and the incidence of ESKD. The prevalence of hematuria was defined as a test result of five or more red blood cells per high power field on urinary sediment. In this analysis, patients with hematuria at the time of renal biopsy were defined as the hematuria group, and those without hematuria were defined as the no-hematuria group.

\section{End point}

The primary end point of the present study was the development of ESKD, which was defined as a requirement of permanent renal replacement therapies. These events were checked from hospital charts or phone calls to a patient or her/his family. As the secondary end point, we evaluated all-cause death in the follow-up period.

\section{Pathological evaluations}

Histological examinations were performed independently by at least two renal pathologists, with differences resolved by consensus. The detailed method of pathological evaluations was previously described. ${ }^{11}$ Briefly, glomerular lesions were classified based on the criteria of the Research Committee of Renal Pathological Society ${ }^{15}$ : class I, characterized by thickening of the glomerular basement membrane as detected by electron microscopy; classes IIa and IIb, mild and severe mesangial expansion, respectively; class III, nodular sclerosis with $<50 \%$ global glomerulosclerosis and class IV, $>50 \%$ global glomerulosclerosis. We did not include class I because the renal tissues were not examined by electron microscopy. Severity of interstitial fibrosis and tubular atrophy (IFTA) was graded as follows: 0, no IFTA; $1,<25 \%$ IFTA; 2, 25\%-50\% IFTA; 3, >50\% IFTA. Vascular lesions were classified by arteriolar hyalinosis and arteriosclerosis (intimal thickening). Arteriolar hyalinosis was graded as follows: 0 , absent; 1 , at least one area; 2, more than one area. The severity of arteriosclerosis was presented as 0 , no intimal thickening; 1 , intimal thickening less than thickness of media and 2: intimal thickening greater than thickness of media. ${ }^{15}$

\section{Statistical analyses}

Categorical variables were expressed as numbers and percentages and were compared with the $\chi^{2}$ test. Continuous variables were expressed as mean $\pm \mathrm{SD}$ or median (IQR). Based on their distribution, continuous variables were compared using the Student's t-test or Wilcoxon rank sum test as appropriate.

Survival curves were obtained using the Kaplan-Meier method and compared by a log-rank test. The Cox proportional hazards model was used to calculate the HR and $95 \%$ CI for the impact of hematuria on ESKD. In the multivariable models, model 1 was adjusted for sex and age; model 2 was adjusted for model 1 plus eGFR, proteinuria, systolic blood pressure and BMI and model 3 was adjusted for model 2 plus pathological findings (glomerular lesion class and IFTA score). In pathological findings, we stratified the glomerular lesion class and IFTA score by IIa-IIb/III-IV and 0-1/2-3, respectively.

Subgroup analyses were conducted in groups stratified by sex, age, proteinuria, eGFR, BMI, systolic blood pressure and pathological findings. In the analyses, we dichotomized age, proteinuria, eGFR, BMI and systolic blood pressure by median values of 59 years, $0.5 \mathrm{~g} /$ day, $58 \mathrm{~mL} /$ $\min / 1.73 \mathrm{~m}^{2}, 23.6 \mathrm{~kg} / \mathrm{m}^{2}$ and $132 \mathrm{~mm} \mathrm{Hg}$, respectively. In each subgroup, we used the same multivariable models (model 3) to assess the association between hematuria and ESKD.

As a sensitivity analysis, we performed the univariable and multivariable Cox proportional hazards models to analyze the association between the prevalence of 
hematuria and the composite end point of ESKD and all-cause death because patients with diabetic nephropathy were at high risk for ESKD, and other events, such as cardiovascular disease.

All analyses were performed using JMP 14.3.0 (SAS Institute, Cary, North Carolina, USA). P values $<0.05$ were considered statistically significant in all analyses except for interaction analyses, which were considered statistically significant when $\mathrm{p} \leq 0.1$.

\section{RESULTS}

Baseline characteristics of the patients with/without

\section{hematuria}

Of the 397 patients with biopsy-proven diabetic nephropathy, 91 (23\%) had hematuria at the time of renal biopsy. In the hematuria group, systolic and diastolic blood pressure, serum creatinine levels and proteinuria were significantly higher, and eGFR was significantly lower than in the no-hematuria group (table 1). Pathological evaluations revealed that the hematuria group had more advanced findings: $59 \%$ of them had class III-IV glomerular lesions, and $61 \%$ of them had IFTA scores of 2-3. Regarding vascular lesions, the arteriolar hyalinosis score was significantly higher in the hematuria group, but the arteriosclerosis score was not statistically significant in either group.

\section{The incidence of ESKD in patients with/without hematuria}

The median follow-up period was 10.1 (95\% CI 8.4 to 11.8) years. During the follow-up period, 44 and 52 patients developed ESKD in the hematuria group and the no-hematuria group, respectively (hematuria group: 69.1/1000 person-years, no-hematuria group: 16.4/1000 person-years). The survival curves showed that the incidence of ESKD was significantly higher in the hematuria group (log-rank, $\mathrm{p}<0.0001$; figure 1$)$. The HR (95\% CI) of hematuria for the incidence of ESKD was 4.11 (95\% CI 2.73 to 6.18 ). The multivariable Cox proportional hazard models revealed the association between hematuria and the incidence of ESKD after adjusting for clinically relevant factors (model 3: adjusted HR 1.64, 95\% CI 1.03 to 2.60; figure 1).

There was a difference in the associations between hematuria and the incidence of ESKD among some of the subgroups (figure 2). Notably, the subgroups of men, proteinuria $\geq 0.5 \mathrm{~g} /$ day and systolic blood pressure $\geq 132 \mathrm{~mm} \mathrm{Hg}$ showed a stronger association between hematuria and ESKD than their opposing subgroups.

\section{The incidence of ESKD and all-cause death in patients with/ without hematuria}

As a sensitivity analysis, we evaluated the composite end point of ESKD and all-cause death. Fifty-three and 90 patients developed ESKD or death in the hematuria group and the no-hematuria group, respectively (hematuria group: 83.3/1000 person-years, no-hematuria group: 28.3/1000 person-years). The survival analyses showed that the incidence of ESKD and death was significantly higher in the hematuria group (log-rank, $\mathrm{p}<0.0001)$, and the crude and adjusted HRs (95\% CIs) of hematuria were 2.93 (2.08 to 4.13 ) and 1.41 (0.96 to 2.08) (model 3), respectively (figure 3 ).

\section{DISCUSSION}

The present study of biopsy-proven diabetic nephropathy showed that patients with hematuria had more advanced pathological findings than those without hematuria. The incidence of ESKD was increased in the patients with hematuria, even after adjusting for clinically relevant factors, including proteinuria and renal pathology. We suggest that the presence of hematuria is a significant risk factor for ESKD in patients with diabetic nephropathy, especially in male patients and patients with comorbid high proteinuria or high blood pressure. The sensitivity analysis showed similar findings, considering deaths before the onset of ESKD.

In our study, the pathological findings revealed that advanced glomerular, interstitial and vascular lesions were observed in the hematuria group (table 1). Over $50 \%$ of the patients in the hematuria group were classified into glomerular lesion classes III-IV (nodular sclerosis or advanced glomerulosclerosis), while many of the patients in the no-hematuria group remained in glomerular lesion class IIa (mild mesangial expansion). In addition, over $60 \%$ of the patients in the hematuria group had $\geq 25 \%$ IFTA. Accumulated evidence showed that severity of glomerular and IFTA lesions were positively associated with the incidence of ESKD in patients with biopsy-proven diabetic nephropathy. ${ }^{17}$ Therefore, it is reasonable to conclude that the presence of hematuria might be associated with a high incidence of ESKD via advanced glomerular and interstitial lesions. However, our study showed a significant association between hematuria and ESKD, even after adjusting for glomerular and interstitial lesions (figure 1). Moreover, in the subgroup analyses, the severity of glomerular and interstitial lesions did not significantly affect the association between hematuria and ESKD (figure 2). A more recent clinical study reported that hematuria in patients with biopsy-proven diabetic nephropathy was associated with advanced renal pathology and a higher risk of ESKD. ${ }^{18}$ However, this study did not demonstrate whether the effect of hematuria on ESKD was independent of renal pathology.

The level of proteinuria was significantly higher in the hematuria group (table 1). A recent study recruited patients with biopsy-proven diabetic nephropathy also showed that the level of proteinuria was higher in the patients with hematuria. ${ }^{18}$ Another study in patients with biopsy-proven diabetic nephropathy reported that the presence of hematuria was associated with the presence of nephrotic syndrome. ${ }^{19}$ Considering the pathological findings in renal biopsy, hematuria accompanied by proteinuria reflected advanced stages of diabetic nephropathy. In our study, the presence of hematuria was significantly associated with an increased incidence 
Table 1 Baseline characteristics of the patients with/without hematuria

\begin{tabular}{|c|c|c|c|c|c|}
\hline & \multirow{2}{*}{$\begin{array}{l}\text { Total } \\
\mathrm{N}=397\end{array}$} & \multirow{2}{*}{$\begin{array}{l}\text { Hematuria } \\
\mathrm{N}=91\end{array}$} & \multirow{2}{*}{$\begin{array}{l}\text { No-hematuria } \\
\mathrm{N}=306\end{array}$} & \multirow{2}{*}{$\begin{array}{l}\text { No. of } \\
\text { missing values }\end{array}$} & \multirow[b]{2}{*}{$P$ value } \\
\hline & & & & & \\
\hline Age, years & $57.7 \pm 11.3$ & $59.1 \pm 10.8$ & $57.3 \pm 11.5$ & 0 & 0.2 \\
\hline Men, n (\%) & $249(63)$ & $65(60)$ & $184(71)$ & 0 & 0.05 \\
\hline $\mathrm{BMI}, \mathrm{kg} / \mathrm{m}^{2}$ & $24.1 \pm 3.8$ & $24.7 \pm 4.0$ & $23.9 \pm 3.7$ & 0 & 0.08 \\
\hline \multicolumn{6}{|l|}{ Blood pressure, mm Hg } \\
\hline Systole & $135 \pm 24$ & $145 \pm 27$ & $132 \pm 22$ & 0 & $<0.0001$ \\
\hline Diastole & $75 \pm 13$ & $78 \pm 15$ & $75 \pm 13$ & 0 & 0.04 \\
\hline Smoking, n (\%) & & & & 0 & 0.4 \\
\hline Never & $154(39)$ & $30(33)$ & $124(41)$ & & \\
\hline Past & $61(15)$ & $14(15)$ & $47(15)$ & & \\
\hline Current & $182(46)$ & $47(52)$ & $135(44)$ & & \\
\hline Diabetic retinopathy, n (\%) & $175(45)$ & $58(65)$ & 117 (39) & 10 & $<0.0001$ \\
\hline \multicolumn{6}{|l|}{ Laboratory findings } \\
\hline Serum creatinine, $\mathrm{mg} / \mathrm{dL}$ & $\begin{array}{l}0.99 \\
(0.70-1.30)\end{array}$ & $\begin{array}{l}1.23 \\
(0.80-1.80)\end{array}$ & $\begin{array}{l}0.90 \\
(0.70-1.20)\end{array}$ & 0 & $<0.0001$ \\
\hline Serum creatinine, $\mu \mathrm{mol} / \mathrm{L}$ & $\begin{array}{l}87.5 \\
(61.9-114.9)\end{array}$ & $\begin{array}{l}108.7 \\
(70.7-159.1)\end{array}$ & $\begin{array}{l}79.6 \\
(61.9-106.1)\end{array}$ & 0 & $<0.0001$ \\
\hline eGFR, $\mathrm{mL} / \mathrm{min} / 1.73 \mathrm{~m}^{2}$ & $\begin{array}{l}57.6 \\
(39.7-80.8)\end{array}$ & $\begin{array}{l}45.4 \\
(29.0-65.7)\end{array}$ & $\begin{array}{l}61.2 \\
(43.4-82.0)\end{array}$ & 0 & $<0.0001$ \\
\hline Proteinuria, g/day & $0.4(0.2-2.5)$ & $3.1(0.5-6.8)$ & $0.3(0.1-1.0)$ & 8 & $<0.0001$ \\
\hline $\mathrm{HbA} 1 \mathrm{c}, \%$ & $8.3 \pm 2.4$ & $7.9 \pm 2.2$ & $8.4 \pm 2.4$ & 36 & 0.1 \\
\hline $\mathrm{HbA} 1 \mathrm{c}, \mathrm{mmol} / \mathrm{mol}$ & $67 \pm 26$ & $63 \pm 24$ & $68 \pm 26$ & 36 & 0.1 \\
\hline \multicolumn{6}{|l|}{ Pathological findings } \\
\hline Glomerular lesion class, n (\%) & & & & 0 & $<0.0001$ \\
\hline Ila & 127 (32) & $12(13)$ & $115(38)$ & & \\
\hline $11 \mathrm{~b}$ & $148(37)$ & $25(27)$ & $123(40)$ & & \\
\hline III & $99(25)$ & $40(44)$ & $59(19)$ & & \\
\hline IV & $23(6)$ & $14(15)$ & $9(3)$ & & \\
\hline IFTA score, n (\%) & & & & 0 & $<0.0001$ \\
\hline 0 & $32(8)$ & $4(4)$ & $28(9)$ & & \\
\hline 1 & $220(55)$ & $31(34)$ & $189(62)$ & & \\
\hline 2 & $66(17)$ & $22(24)$ & $44(14)$ & & \\
\hline 3 & $79(20)$ & $34(37)$ & $45(15)$ & & \\
\hline Arteriolar hyalinosis score, $\mathrm{n}(\%)$ & & & & 0 & $<0.02$ \\
\hline 0 & $55(14)$ & $7(8)$ & $48(16)$ & & \\
\hline 1 & $120(30)$ & $22(24)$ & $98(32)$ & & \\
\hline 2 & $222(56)$ & $62(68)$ & $160(52)$ & & \\
\hline Arteriosclerosis score, $\mathrm{n}(\%)$ & & & & 31 & 0.9 \\
\hline 0 & $78(21)$ & $17(20)$ & $61(22)$ & & \\
\hline 1 & $123(34)$ & $30(36)$ & $93(33)$ & & \\
\hline 2 & $165(45)$ & $36(43)$ & $129(46)$ & & \\
\hline
\end{tabular}

BMI, body mass index; eGFR, estimated glomerular filtration rate; HbA1c, hemoglobin A1c; IFTA, intestinal fibrosis and tubular atrophy.

of ESKD, even after adjusting for proteinuria. We also showed that hematuria was more strongly associated with ESKD in the subgroup of proteinuria $\geq 0.5 \mathrm{~g} /$ day (figure 2). These results suggest that hematuria and proteinuria had a synergistic effect on CKD progression in patients with diabetic nephropathy. Similar findings were reported in IgA nephropathy. In the study, patients with IgA nephropathy with hematuria accompanied by 


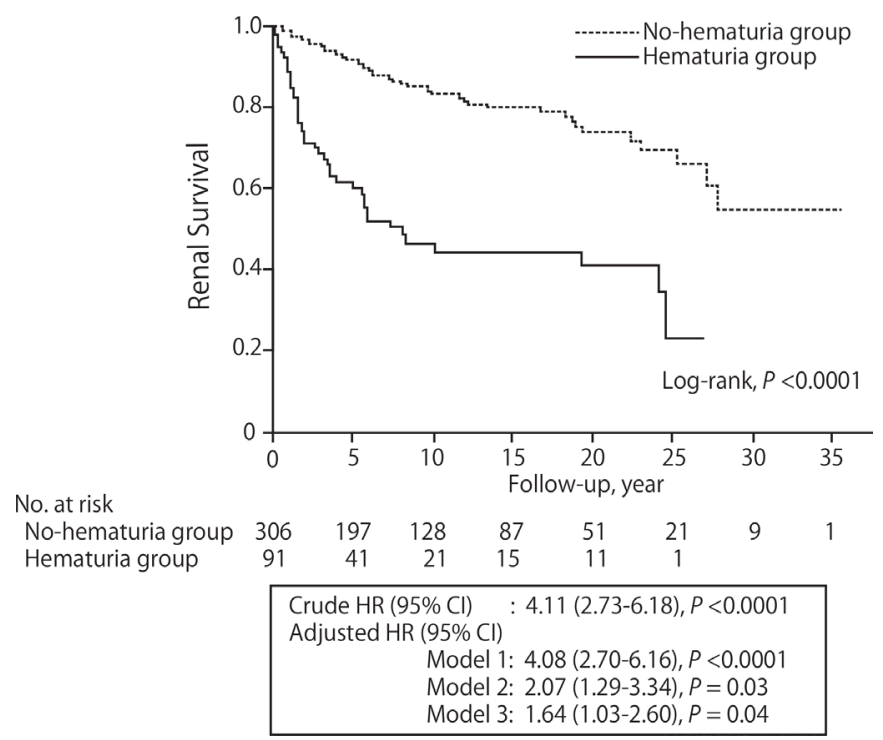

Figure 1 The incidence of ESKD in patients with/without hematuria. The survival curves showed that the incidence of ESKD was significantly higher in the hematuria group (logrank, $\mathrm{p}<0.0001)$. The crude HR $(95 \% \mathrm{Cl})$ of hematuria for the incidence of ESKD was 4.11 (2.73 to 6.18). The association remains significant after adjusting for clinically relevant factors. Model 1 was adjusted for sex and age. Model 2 was adjusted for model 1 plus eGFR, proteinuria, systolic blood pressure and BMI. Model 3 was adjusted for model 2 plus pathological findings (glomerular lesion class and IFTA score). BMI, body mass index; eGFR, estimated glomerular filtration rate; ESKD, end-stage kidney disease; IFTA, interstitial fibrosis and tubular atrophy.

proteinuria had a significantly higher incidence of ESKD than the others. ${ }^{20}$

In this study, the subgroup analyses suggested that the prevalence of hematuria was associated with an increased incidence of ESKD in men, not in women (figure 2). In the general population, the prevalence of asymptomatic hematuria is higher in women than men at all age groups, ${ }^{21} 22$ and as a result, the prevalence of accidental

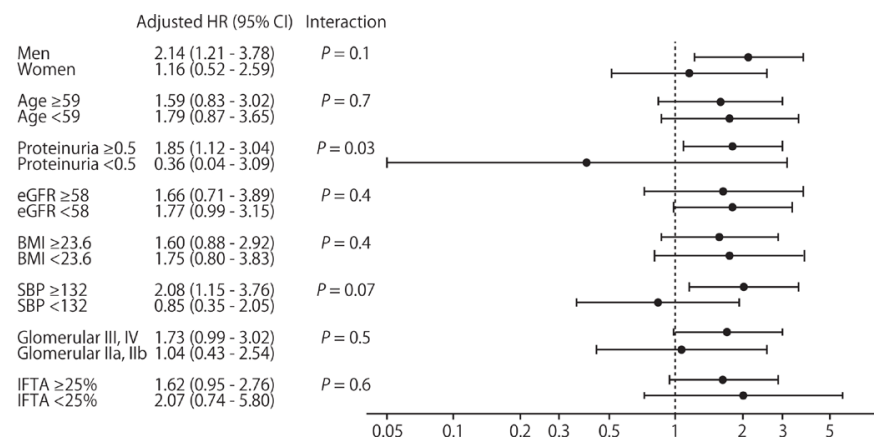

Figure 2 Subgroup analyses. We dichotomized age, proteinuria, eGFR, BMI and SBP by median values. In each subgroup, we used the multivariable Cox proportional hazard model (model 3) to assess the association between hematuria and ESKD. BMI, body mass index; eGFR, estimated glomerular filtration rate; ESKD, end-stage kidney disease; IFTA, intestinal fibrosis and tubular atrophy; SBP, systolic blood pressure.

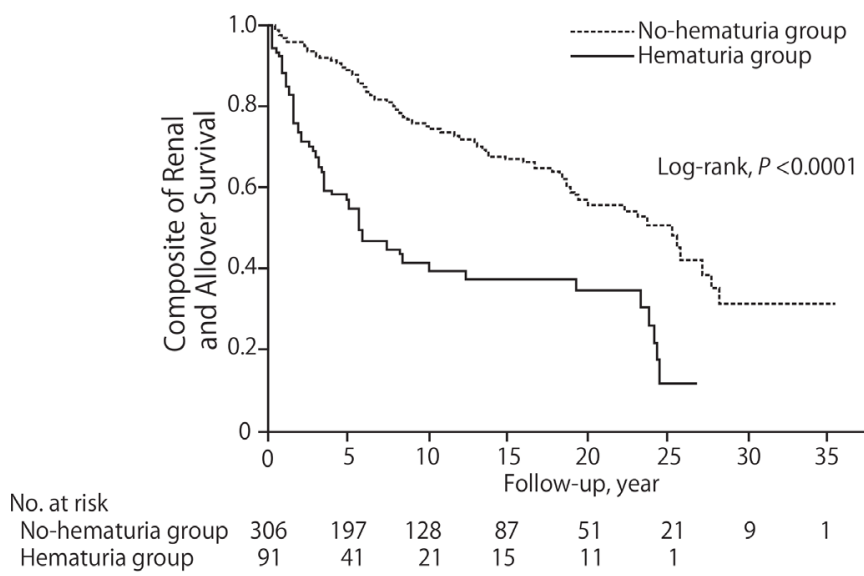

\begin{aligned} \hline Crude HR $(95 \% \mathrm{Cl}) \quad: 2.93(2.08-4.13), P<0.0001 \\$ Adjusted HR $(95 \% \mathrm{Cl}) \\$ Model 1: $2.74(1.93-3.88), P<0.0001 \\$ Model 2: $1.68(1.13-2.49), P=0.01 \\$ Model 3: $1.41(0.96-2.08), P=0.08\end{aligned}$

Figure 3 The incidence of ESKD and all-cause death in patients with/without hematuria. As a sensitivity analysis, we evaluated the composite end point of ESKD and all-cause death. The survival curves showed that the incidence of ESKD and death was significantly higher in the hematuria group (log-rank, $\mathrm{p}<0.0001$ ), and the crude and adjusted HRs $(95 \% \mathrm{Cl})$ of hematuria were 2.93 (2.08 to 4.13$)$ and 1.41 (0.96 to 2.08 ) (model 3), respectively. Model 1 was adjusted for sex and age. Model 2 was adjusted for model 1 plus eGFR, proteinuria, systolic blood pressure and BMI. Model 3 was adjusted for model 2 plus pathological findings (glomerular lesion class and IFTA score). BMI, body mass index; eGFR, estimated glomerular filtration rate; ESKD, end-stage kidney disease; IFTA, intestinal fibrosis and tubular atrophy.

hematuria unrelated to diabetic nephropathy is also considered to be higher in women. Therefore, the presence of hematuria in female patients might not be associated with the incidence of ESKD. Similar findings were reported in a previous study. ${ }^{18}$

Our long-term follow-up study revealed, for the first time, that the presence of microscopic hematuria was associated with the incidence of ESKD in patients with biopsy-proven diabetic nephropathy, independent of renal pathology. However, our study has several limitations. First, the study sample size was small, and only 96 patients developed ESKD in total. We made the necessary adjustments to the multivariable analyses, but we needed to interpret the results carefully, especially in the subgroup analyses. Second, we recruited only patients with type 2 diabetes who underwent renal biopsy. The patients' characteristics of our cohort might differ from general population with diabetes. For example, since hematuria in patients with diabetes is one of the indications of renal biopsy, ${ }^{5}$ the frequency of hematuria in our cohort was considered to be higher than in general patients with diabetes. Therefore, it remains uncertain whether these findings are applicable to all patients with diabetes. Third, we could not exclude patients with transient hematuria. Transient microscopic hematuria is often observed in the general population $(34 \%)^{23}$; 
however, the rate of transient hematuria is low in patients with advanced diabetic nephropathy $(12 \%) .{ }^{24}$ Thus, transient hematuria had only a small influence on the present study. Finally, we could not evaluate the duration of diabetes. Duration of diabetes is a key factor in the diabetic state, ${ }^{25}$ and associated with the development of diabetic nephropathy. ${ }^{26} \mathrm{~A}$ previous report indicated that a long duration of diabetes was associated with the presence of hematuria in patients with biopsy-proven diabetic nephropathy. ${ }^{19}$ On the other hand, another report showed no difference in the duration of diabetes among glomerular lesions in patients with biopsy-proven diabetic nephropathy. ${ }^{27}$ The duration of diabetes seems to be an important factor in diabetic nephropathy progression, and we should take into account its influence on hematuria and renal function.

In conclusion, the presence of hematuria has been generally considered a clinical sign of non-diabetic kidney disease in patients with diabetes; however, our present study demonstrated that microscopic hematuria in diabetic nephropathy indicates advanced pathological findings in renal biopsy. Our study showed that the patients with hematuria have a high incidence of ESKD, independent of proteinuria and renal pathology. We should consider that the presence of hematuria is a risk factor for ESKD in diabetic nephropathy.

Acknowledgements The authors would like to thank T. Kanki, M. Nishimoto, M. Murashima, M. Iwano, H. Shiiki, H. Ymada, M. Kanauchi and K. Dohi for their roles in constructing the study cohort. The authors would also like to thank Editage ( www.editage.com) for English language editing.

Contributors S0 designed the study, researched data and wrote the manuscript. K-iS contributed to constructing the study cohort, researched data and reviewed/ edited the manuscript. SO and K-iS contributed equally to this work. MM, KM, $\mathrm{RF}$ and KTa contributed to constructing the study cohort and the discussion, and reviewed the manuscript. ME contributed to constructing the study cohort, researched data and reviewed/edited the manuscript. YA and YS contributed to constructing the study cohort and the discussion and reviewed the manuscript. KTs contributed to constructing the study cohort and reviewed/edited the manuscript. $\mathrm{K}$-iS is the guarantor of this work, who takes full responsibility for this work.

Funding This work was supported in part by JSPS KAKENHI Grant Number 15K19462.

Competing interests S0, K-iS, MM, KM, RF, KTa and ME have nothing to disclose. YA reports grants and personal fees from Sumitomo Dainippon Pharma; grants from Japanese Red Cross Society Nara Red Cross Blood Center and Kissei Pharma; personal fees from Nippon Boehringer Ingelheim, outside the submitted work. YS reports grants and personal fees from Daiichi Sankyo, Mitsubishi Tanabe Pharma, Novartis Pharma and Otsuka Pharma; grants from Ono Pharma, Takeda Pharma, Bristol-Myers Squibb, Astellas Pharma, Shionogi, Teijin, Bayer, Actelion Pharma, Kirin, Kowa, Sumitomo Dainippon Pharma, Amgen Astellas BioPharma, EP-CRSU, Roche Diagnostics and Terumo, outside the submitted work. KTs reports grants and personal fees from Baxter, Bayer and Kirin; grants from Terumo; personal fees from Chugai Pharma, Asteras Pharma, Mitsubishi Tanabe Pharma, Kissei Pharma, Sumitomo Dainippon Pharma and JCR Pharma, outside the submitted work.

Patient consent for publication Not required.

Ethics approval The study protocol was approved by the Nara Medical University Ethics Committee (2005-18) and registered in the University Hospital Medical Information Network (UMIN) clinical trial registry (UMIN000031121). Informed consent was obtained from each patient.

Provenance and peer review Not commissioned; externally peer reviewed.

Data availability statement Data are available on reasonable request. Please contact the corresponding author.
Open access This is an open access article distributed in accordance with the Creative Commons Attribution Non Commercial (CC BY-NC 4.0) license, which permits others to distribute, remix, adapt, build upon this work non-commercially, and license their derivative works on different terms, provided the original work is properly cited, appropriate credit is given, any changes made indicated, and the use is non-commercial. See: http://creativecommons.org/licenses/by-nc/4.0/.

ORCID iD

Ken-ichi Samejima http://orcid.org/0000-0001-8273-5155

\section{REFERENCES}

1 Moreno JA, Yuste C, Gutiérrez E, et al. Haematuria as a risk factor for chronic kidney disease progression in glomerular diseases: a review. Pediatr Nephrol 2016;31:523-33.

2 Vivante A, Afek A, Frenkel-Nir Y, et al. Persistent asymptomatic isolated microscopic hematuria in Israeli adolescents and young adults and risk for end-stage renal disease. JAMA 2011;306:729-36.

3 Kido R, Shibagaki Y, Iwadoh K, et al. Persistent glomerular hematuria in living kidney donors confers a risk of progressive kidney disease in donors after heminephrectomy. Am J Transplant 2010;10:1597-604.

4 Yuste C, Rubio-Navarro A, Barraca D, et al. Haematuria increases progression of advanced proteinuric kidney disease. PLoS One 2015;10:e0128575

5 American Diabetes Association. 11. Microvascular Complications and Foot Care: Standards of Medical Care in Diabetes-2020. Diabetes Care 2020;43:S135-51.

6 Zhou J, Chen X, Xie Y, et al. A differential diagnostic model of diabetic nephropathy and non-diabetic renal diseases. Nephrol Dial Transplant 2008:23:1940-5.

7 Chong Y-B, Keng T-C, Tan L-P, et al. Clinical predictors of non-diabetic renal disease and role of renal biopsy in diabetic patients with renal involvement: a single centre review. Ren Fail 2012;34:323-8.

8 Tuttle KR, Bakris GL, Bilous RW, et al. Diabetic kidney disease: a report from an ADA consensus conference. Diabetes Care 2014;37:2864-83.

9 Shimizu M, Furuichi K, Toyama T, et al. Long-Term outcomes of Japanese type 2 diabetic patients with biopsy-proven diabetic nephropathy. Diabetes Care 2013;36:3655-62.

10 Matsumura N, Hanatani M, Nishino T, et al. [The clinico-pathological significance of hematuria in diabetics]. Nihon Jinzo Gakkai Shi 1994;36:1036-45.

11 Morimoto K, Matsui M, Samejima K, et al. Renal arteriolar hyalinosis, not intimal thickening in large arteries, is associated with cardiovascular events in people with biopsy-proven diabetic nephropathy. Diabet Med 2020. doi:10.1111/dme.14301. [Epub ahead of print: 10 Apr 2020].

12 Imai E, Horio M, Nitta K, et al. Estimation of glomerular filtration rate by the MDRD study equation modified for Japanese patients with chronic kidney disease. Clin Exp Nephrol 2007;11:41-50.

13 Matsuo S, Imai E, Horio M, et al. Revised equations for estimated GFR from serum creatinine in Japan. Am J Kidney Dis 2009;53:982-92.

14 Committee of the Japan Diabetes Society on the Diagnostic Criteria of Diabetes Mellitus, Seino Y, Nanjo K, et al. Report of the Committee on the classification and diagnostic criteria of diabetes mellitus. J Diabetes Investig 2010;1:212-28.

15 Tervaert TWC, Mooyaart AL, Amann K, et al. Pathologic classification of diabetic nephropathy. J Am Soc Nephrol 2010;21:556-63.

16 Oh SW, Kim S, Na KY, et al. Clinical implications of pathologic diagnosis and classification for diabetic nephropathy. Diabetes Res Clin Pract 2012;97:418-24.

17 Stefan G, Stancu S, Zugravu A, et al. Histologic predictors of renal outcome in diabetic nephropathy: beyond renal pathology Society classification. Medicine 2019;98:e16333.

$18 \mathrm{Wu} \mathrm{Y}$, Zhang J, Wang $\mathrm{Y}$, et al. The association of hematuria on kidney clinicopathologic features and renal outcome in patients with diabetic nephropathy: a biopsy-based study. J Endocrinol Invest 2020;43:1213-20.

19 Akimoto T, Ito C, Saito O, et al. Microscopic hematuria and diabetic glomerulosclerosis--clinicopathological analysis of type 2 diabetic patients associated with overt proteinuria. Nephron Clin Pract 2008;109:c119-26.

20 Sevillano AM, Gutiérrez E, Yuste C, et al. Remission of hematuria improves renal survival in IgA nephropathy. J Am Soc Nephrol 2017:28:3089-99.

21 Yamagata K, Yamagata Y, Kobayashi M, et al. A long-term follow-up study of asymptomatic hematuria and/or proteinuria in adults. Clin Nephrol 1996;45:281-8. 
22 Ishida K, Ishida H, Yamagata K, et al. [Significance and the current status of urinalysis and serum creatinine determination for adults (with special reference to the health examination data at Ibaragi Prefecture]. Nihon Naika Gakkai Zasshi 2001;90:1199-206.

23 Loo RK, Lieberman SF, Slezak JM, et al. Stratifying risk of urinary tract malignant tumors in patients with asymptomatic microscopic hematuria. Mayo Clin Proc 2013;88:129-38.

24 Okada T, Nagao T, Matsumoto $\mathrm{H}$, et al. Clinical significance of microscopic haematuria in diabetic nephropathy in type 2 diabetes patients with overt proteinuria. Nephrology 2013;18:563-8.
25 Kendall DM, Cuddihy RM, Bergenstal RM. Clinical application of incretin-based therapy: therapeutic potential, patient selection and clinical use. Am J Med 2009;122:S37-50.

26 Tone A, Shikata K, Matsuda M, et al. Clinical features of non-diabetic renal diseases in patients with type 2 diabetes. Diabetes Res Clin Pract 2005;69:237-42.

27 Okada T, Nagao T, Matsumoto H, et al. Histological predictors for renal prognosis in diabetic nephropathy in diabetes mellitus type 2 patients with overt proteinuria. Nephrology 2012;17:68-75. 